\title{
Magnetic Structure in UIrAl
}

\author{
K. Proke $\check{S}^{a}$, J. Prchal ${ }^{b}$, V. SechovskÝ ${ }^{b}$ And A.V. Andreev ${ }^{c}$ \\ ${ }^{a}$ Hahn-Meitner-Institute, SF-2, Glienicker Str. 100, 14109 Berlin, Germany \\ ${ }^{b}$ Charles University, Faculty of Mathematics and Physics \\ Department of Condensed Matter Physics \\ Ke Karlovu 5, 12116 Prague, Czech Republic \\ ${ }^{c}$ Institute of Physics, ASCR, Na Slovance 2, 18221 Prague, Czech Republic
}

\begin{abstract}
By means of neutron diffraction on a hexagonal UIrAl single crystal we find that this system orders ferromagnetically at low temperatures with magnetic moments of $0.93(10) \mu_{\mathrm{B}}$ aligned along the $c$ axis. The Curie temperature $T_{\mathrm{C}}=65(5) \mathrm{K}$ determined from the diffraction experiment is in good agreement with bulk magnetic measurements.
\end{abstract}

PACS numbers: $75.25 .+\mathrm{z}$

\section{Introduction}

UIrAl crystallizing in the hexagonal $\mathrm{ZrNiAl}$ structure belongs to a large group of uranium intermetallic compounds UTX ( $\mathrm{T}=$ transition metal, $\mathrm{X}=p$ element) intensively studied in last decades [1]. Magnetization studies performed on the polycrystalline samples revealed that UIrAl is a ferromagnet with the Curie temperature $T_{\mathrm{C}}=64 \mathrm{~K}$ and the spontaneous magnetic moment $M_{\mathrm{s}}=0.94 \mu_{\mathrm{B}}$ $[2,3]$. The well known layered hexagonal crystal structure of the ZrNiAl-type implies very anisotropic physical properties. Comparison of the magnetization curves measured on free and randomly fixed powders pointed to a uniaxial magnetocrystalline anisotropy [3]. Single-crystal studies [4, 5] revealing $T_{\mathrm{C}}=62 \mathrm{~K}$ and $M_{\mathrm{s}}=0.96 \mu_{\mathrm{B}}$ as well as the $c$-axis as the easy magnetization direction confirm the results of studies on polycrystals. The huge uniaxial anisotropy with an anisotropy field exceeding $200 \mathrm{~T}$ leads to characteristic hysteresis properties dictated by very narrow domain walls. In this paper we report on results of magnetic bulk and magnetoresistance measurements combined with a neutron diffraction experiment using a high quality UIrAl single crystal.

The magnetization curve and the hysteresis loop measured at $4.2 \mathrm{~K}$ with the field along the $c$ axis reported in $[4,5]$ shows almost rectangular profile and a $100 \%$ remanent magnetization. Such a behavior, which is caused by a narrow-domainwall pinning, is typical of ferromagnets $(\mathrm{F})$ with a very large magnetic anisotropy energy that is comparable or exceeding the exchange interaction energy. In Ref. [4] it is shown that the pinning model is applicable also for UPtAl. Typical of the 
model is a fast decrease in the hysteresis and the coercive field with increasing temperature due to thermal activation.

Nevertheless, an interpretation that the ground state is of UIrAl is antiferromagnetic $(\mathrm{AF})$, and the magnetization increases due to a change in a microscopic arrangement of magnetic moments across a metamagnetic-like transition cannot be excluded. Hysteretic effects connected with the first-order transition stabilizes the ferromagnetic state in zero and even in some negative fields. Clearly, the magnetization study alone cannot distinguish between the two cases because the movement of a monoatomic domain wall means in fact a flip of magnetic moments like at a metamagnetic transition. Among the UTX compounds exhibiting similar hysteretic properties, both $\mathrm{F}$ and $\mathrm{AF}$ ground state were observed. The isostructural UPtAl [6] and UCoGa [7] are ferromagnets whereas UNiGa is an antiferromagnet [8]. For this reason, we checked the ground state of UIrAl by neutron diffraction experiment.

\section{Experimental}

The single crystal of UIrAl has been grown by a modified Czochralski method from a stoichiometric melt. Details are given in [4].

The magnetization was measured in the temperature range $2-70 \mathrm{~K}$ in a vibrating sample magnetometer in fields up to $14 \mathrm{~T}$. For measurements of virgin magnetization curves and hysteresis loop the sample was thermally demagnetized at high temperatures and then cooled down in zero field. The longitudinal magnetoresistivity at low temperatures has been measured by a standard four-point method in fields up to $14 \mathrm{~T}$ applied along the $c$ axis.

The neutron diffraction experiment was performed on the E4 double-axis diffractometer installed at the Berlin Neutron Scattering Center with an incident neutron wavelength of $2.44 \AA$. The single crystal with approximate dimensions $2 \times 2 \times 3 \mathrm{~mm}^{3}$ was first oriented using the X-ray Laue technique and mounted with its $b$ axis perpendicular to the scattering plane. We have collected two identical data sets, one in the paramagnetic state of UIrAl, at $80 \mathrm{~K}$ and other at the lowest temperature achievable with the standard orange-type cryostat, at $1.8 \mathrm{~K}$. The individual scan profiles were collected typically $40 \mathrm{~min}$ each and analyzed by fitting to a Gaussian profile. The crystallographic and magnetic structures were determined by using a set of computer codes WinPLOTR [9]. Correction for Ir absorption has been applied. Appropriate scattering lengths and the standard $\mathrm{U}^{3+}$ magnetic form factor were used in the refinement. Moreover, we have followed intensity of few reflections as a function of temperature.

\section{Results and discussion}

By refinement of the data set at $80 \mathrm{~K}$, above the ordering temperature, we have verified that UIrAl crystallizes in the hexagonal ZrNiAl-type of structure 
with lattice parameters $a=6.929 \AA, c=4.010 \AA$. The free position parameters of $\mathrm{U}$ and $\mathrm{Al}$ were found to be $x_{\mathrm{U}}=0.579(1)$ and $x_{\mathrm{Al}}=0.243(5)$.

At temperatures below $70 \mathrm{~K}$, an additional scattering intensity is observed on the top of seven Bragg nuclear reflections and no new reflections have been seen. The temperature dependence of the integrated intensity of the (100) reflection that contains the highest magnetic contribution normalized to the limiting intensity extrapolated to $0 \mathrm{~K}$ is presented in Fig. 1. The comparison with the temperature dependence of the square of spontaneous magnetization demonstrates a reasonable agreement between the macroscopic and microscopic magnetic data. The magnetic phase transition from our neutron experiment can be estimated as 65 (5) K.

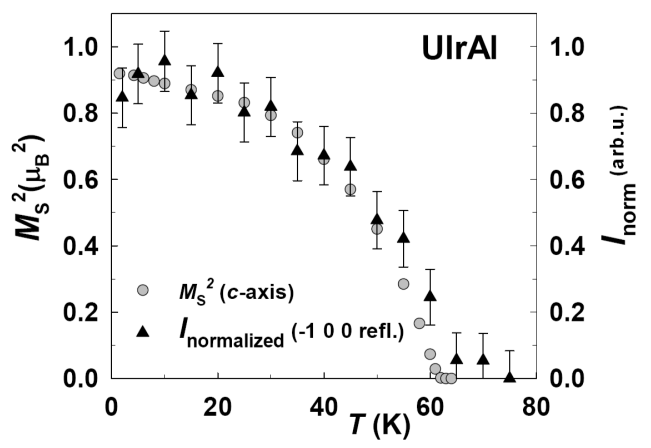

Fig. 1. Temperature dependence of the normalized intensity of the (100) reflection that is of magnetic origin in comparison with the square of magnetization along the $c$-axis.

The magnetic unit cell has thus the identical size as the crystallographic one. This result, however, still does not rule out an AF ordering in UIrAl since the crystallographic unit cell of UIrAl contains three magnetic moments. The symmetry analysis for the given $k$ and the $\mathrm{ZrNiAl}$ type of structure is given in [8]. According to the analysis the moments can be either confined within the basal plane and AF coupled with each other or ferromagnetically aligned along the $c$ axis. The integrated-intensities difference between the ordered $(T=1.8 \mathrm{~K})$ and paramagnetic state $(T=80 \mathrm{~K})$ has been used for the fitting to all models allowed by symmetry. It became clear that the best agreement has been found for the ferromagnetic model. For such a model, in which the only variable parameter has been the magnitude of $\mathrm{U}$ magnetic moments, we have obtained $\chi^{2}=2.01$. All the other models could not account correctly for the observed intensity ratio between (100) and (200) reflections and lead to more than six times larger $\chi^{2}$.

The magnitude of the magnetic moments located on the U sites was found to be $\mu_{\mathrm{U}}=0.93(10) \mu_{\mathrm{B}}$ that is in very good agreement with magnetization data of 0.94 and $0.96 \mu_{\mathrm{B}}$, respectively.

An independent indication that the abrupt increase in magnetization is not caused by a change in the magnetic structure can be found in an absence of any 


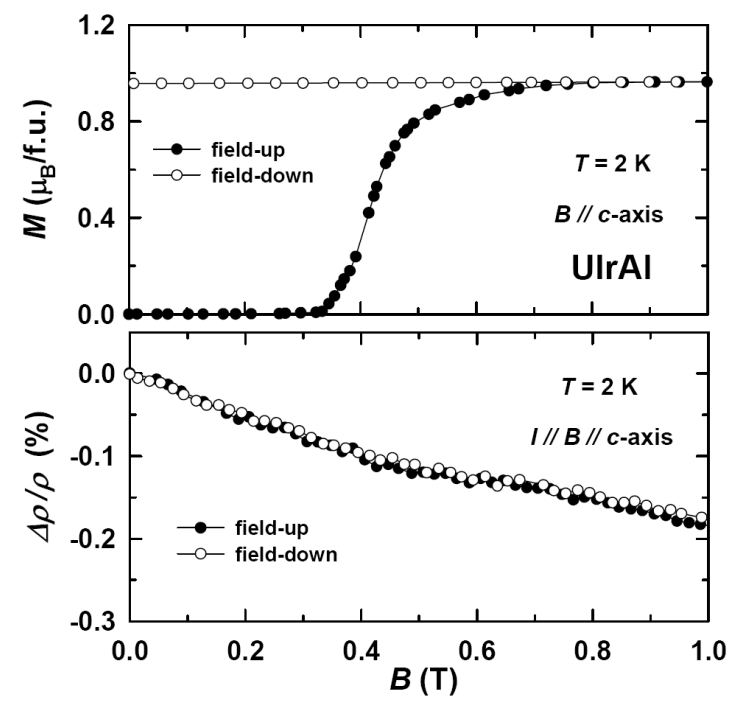

Fig. 2. Low-field portion of magnetization and longitudinal magnetorestivity curves of a UIrAl single crystal in field applied along the $c$-axis at $2 \mathrm{~K}$.

significant magnetoresistance anomaly shown in Fig. 2. For instance, in the case of UNiGa, the resistivity drops by $90 \%$ across the $\mathrm{AF}-\mathrm{F}$ transition [8].

\section{Acknowledgments}

This work is a part of the research plan MSM 0021620834 that is financed by the Ministry of Education of the Czech Republic.

\section{References}

[1] V. Sechovský, L. Havela, in: Handbook of Magnetic Materials, Vol. 11, Ed. K.H.J. Buschow, Elsevier, Amsterdam 1998, p. 1.

[2] A.V. Andreev, M.I. Bartashevich, Phys. Met. Metallogr. 62, 50 (1986).

[3] V. Sechovský, L. Havela, P. Nozar, E. Brück, F.R. de Boer, A.A. Menovsky, K.H.J. Buschow, A.V. Andreev, Physica B 163, 103 (1990).

[4] A.V. Andreev, J. Alloys Comp. 336, 77 (2002).

[5] A.V. Andreev, N.V. Mushnikov, F. Honda, V. Sechovský, P. Javorský, T. Goto, J. Magn. Magn. Mater. 272-276, E337 (2004).

[6] A.V. Andreev, M. Diviš, P. Javorský, K. Prokeš, V. Sechovský, J. Kuneš, Y. Shiokawa, Phys. Rev. B 64, 144408 (2001).

[7] A. Purwanto, R.A. Robinson, K. Prokeš, H. Nakotte, F.R. de Boer, L. Havela, V. Sechovský, N.C. Tuan, Y. Kergadallan, J.C. Spirlet, J. Rebizant, J. Appl. Phys. 76, 7040 (1994).

[8] K. Prokeš, E. Brück, F.R. de Boer, M. Mihalik, A.A. Menovsky, P. Burlet, J.M. Mignot, L. Havela, V. Sechovský, J. Appl. Phys. 79, 6396 (1996).

[9] T. Roisnel, J. Rodriguez-Carvajal, WinPLOTR, version July 2006. 